\title{
Laparoscopy versus laparotomy for the treatment of ovarian cysts in pregnancy: should we change our conventional way of practice?
}

Received: 28 December 2004/ Accepted: 15 March 2005/Published online: 21 May 2005

(C) Springer-Verlag Berlin / Heidelberg 2005

\begin{abstract}
Pregnant women may require nonobstetricrelated abdominopelvic surgery. Traditionally, a laparotomy has been the preferred approach. Recent data suggest that this method should be reviewed in light of developments in minimal access surgical techniques. This article compares both approaches and, in particular, discusses the use of laparoscopy in the 2nd and 3rd trimesters of pregnancy.
\end{abstract}

Keywords Laparoscopy $\cdot$ Pregnancy $\cdot$ Ovarian cyst

\section{Introduction}

Laparotomy has been the standard technique when surgery is required during the 2 nd and 3 rd trimesters of pregnancy. Common indications include acute abdomen, appendicitis, ovarian or adnexal pathology, gallbladder disease, among others. Laparotomy for ovarian pathology (cystectomy or oophorectomy) is one of the procedures gynaecologists become all too familiar with during their training years and beyond.

\section{Case study}

The following is an example of a clinical case report on the typical management of such a situation in pregnancy. A 22-year-old primigravida presented with a $5-\mathrm{cm}$ in diameter right ovarian cyst on routine ultrasound scanning at 10 weeks' gestation. The sonographic

\footnotetext{
C. Panayotidis $(\varangle) \cdot$ J. Martin

Department of Obstetrics and Gynaecology,

South Manchester University Hospitals NHS Trust,

Wythenshawe, Manchester, UK

E-mail: costapan@hotmail.com

M. Lim

Department of Obstetrics and Gynaecology,

Queenspark Hospital, Blackburn, Manchester, UK
}

appearances were consistent with a mature cystic teratoma. The woman was asymptomatic at the time. On a repeat scan at 20 weeks, the cyst had increased to $7 \mathrm{~cm}$. One week later, the woman presented with an acute abdomen with clinical signs highly suggestive of an ovarian accident. A midline laparotomy was performed, and a right ovarian cystectomy with conservation and reconstruction of the right ovary was done (Figs. 1, 2, and 3). The postoperative period was uneventful. No tocolytics were required, ultrasound scanning of the foetus verified its well-being, and the patient was discharged home after 7 days. Her pregnancy continued to term and ended in a normal vaginal delivery.

This case illustrates the common practice of managing ovarian pathology in pregnancy. Informed consent is necessary, particularly when it concerns operative and postoperative complications and the risk of miscarriage or premature labour. However, in view of advances in minimal access surgical techniques and their safety over the past 10 years, a different approach is now feasible. With careful selection of patients, such cases can be treated laparoscopically, and many reports have emerged suggesting that the laparoscopic approach may be as safe as laparotomy with fewer adverse effects for the patient.

\section{Discussion}

Background

Ovarian tumours in pregnancy are estimated to have an incidence of 1 in 1,500 pregnancies, with simple serous cysts being the most common type, followed by dermoid cysts. Ovarian malignancy is rare in this age-related population of women and occurs at an incidence of only 1 in 25,000 pregnancies [1,2].

The direct effects of ovarian tumours vary from causing miscarriage or preterm labour to causing pressure-type symptoms from complicated large tumours. Foetal malpresentations, nonengagement of the foetal 


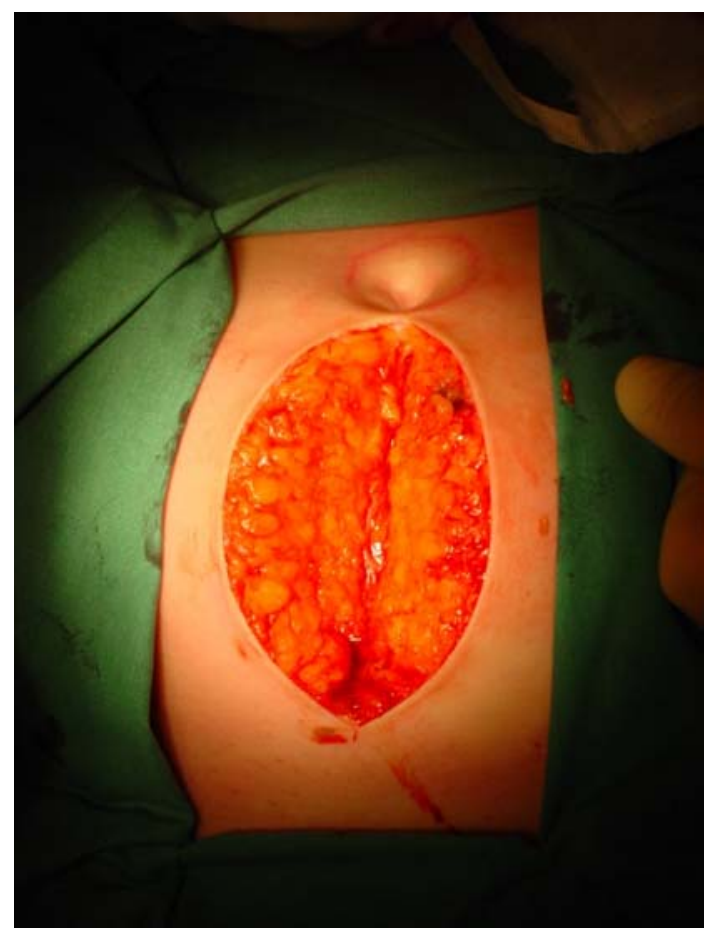

Fig. 1 Median laparotomy

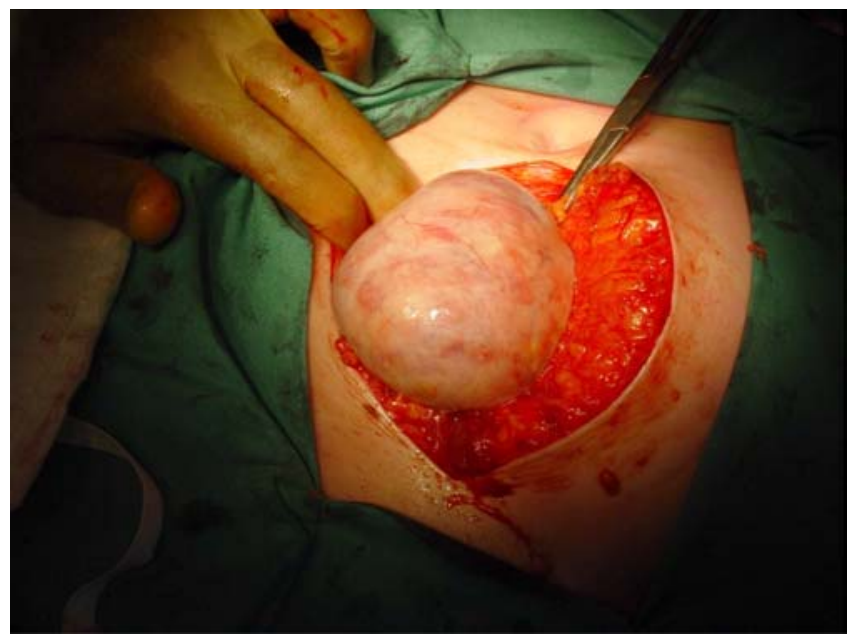

Fig. 2 Dermoid cyst exposed

head, and obstructed labour are obstetric complications associated with these tumours. Ovarian torsion is the most common type of complication when these tumours lie above the pelvic brim, and, due to abdominal wall laxity, its occurrence is more common during the puerperium than in pregnancy because the larger intraabdominal space created after birth affords greater mobility to the enlarged ovary. Other complications include ovarian cyst rupture, haemorrhage, and infection. Rapid growth of the cyst may suggest malignancy [3]. Management depends on the size of the cyst, its rapidity of growth, its ultrasonic appearance, the

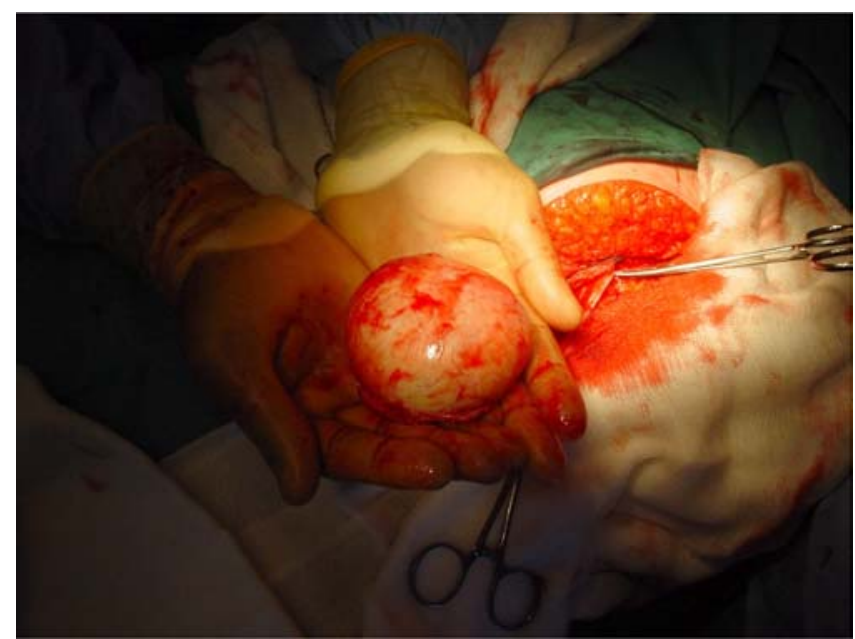

Fig. 3 Cystectomy

presence or absence of symptoms, and the stage of pregnancy. Cysts less than $5-6 \mathrm{~cm}$ in diameter and without symptoms can be treated conservatively and followed up with serial ultrasound scans. They often resolve spontaneously [4]. Larger cysts or those that are symptomatic may need removal. The optimal time for an elective surgical intervention appears to be in the 2 nd trimester between 16 and 20 weeks.

On the other hand, emergencies can happen at any time. Emergency surgery accounts for more foetal compromise and foetal loss [3]. Foetal monitoring at the time of intervention does not seem to modify the foetal prognosis because these emergency cases tend to happen in the 1st trimester. Ovarian tumour sizes tend to be significantly larger in emergency cases, since tumours of less than $4-5 \mathrm{~cm}$ usually are not associated with acute symptoms requiring emergency surgery [3].

\section{Open surgery approach}

Most consultants in the United Kingdom use the classical approach to a laparotomy. Depending on the size and location of the ovarian tumour, a Pfannenstiel, paramedian, or midline incision is selected. The patient is thoroughly counselled about the operation's associated risks to her and to the pregnancy. General anaesthesia risks and premature labour could be associated with the operation, but there have been no randomised controlled trials to suggest a direct link. Tocolysis and long-term antibiotic coverage have been used in the past but are not used in routine practice. Maximum effort should be made to avoid rupturing the ovarian cyst. Dermoid intracystic contents are highly irritating to the peritoneum and may result in chemical peritonitis and adhesions. The uncomplicated postoperative period following a Pfannenstiel incision is 5 days and between 6 and 8 days after a midline incision. The abdominal incision is closed carefully to 
avoid infections or wound dehiscence, as the abdominal volume will continue to increase with the ongoing pregnancy, exerting stress on the healing wound in the months ahead.

\section{Laparoscopic approach}

The most commonly reported laparoscopic operation in pregnancy is cholecystectomy [5, 6]. Other laparoscopic procedures include oophorectomies, ovarian cystectomies, appendicectomies, and management of ectopic pregnancies [5]. Performing a surgical intervention on an ovarian tumour in pregnancy via the laparoscopic route as opposed to the more conventional laparotomy is still controversial. In the hands of an experienced surgeon, the laparoscopic approach may have numerous advantages, especially in a pregnant patient; these advantages may include decreased postoperative morbidity, less pain, lower analgaesia requirements, and earlier recovery to normal bowel function. In turn, this leads to a shorter hospital stay and earlier return to normal activity and mobility, which is essential in preventing thromboembolic disease in pregnancy [5].

\section{Consequences for the pregnancy and foetus}

Haemodynamic changes during laparoscopic surgery in pregnancy are similar to those observed in the nonpregnant patient. The occurrence of miscarriage, premature labour, or foetal demise appears to be related to the underlying pathology, independent of the operative intervention [7]. Two studies, one of which looked into the operative complications and obstetric outcomes between laparoscopy and laparotomy (192 laparoscopies versus 197 laparotomies) [8] and the other of which surveyed surgeons' experiences with laparoscopy in pregnancy (413 cases analysed) [9], have generally concluded that the laparoscopic approach appears to be as safe as laparotomy in pregnancy. Operative and postoperative maternal complications, miscarriages, congenital malformations, and newborn long-term outcomes seem not to differ from what has been recorded with laparotomy (39 laparoscopies versus 54 laparotomies) [10, 11]. Surgery during the 1 st or 2 nd trimester is not associated with significant preterm labour, foetal loss, or teratogenic risk. Surgery during the 3rd trimester is, however, associated with preterm labour but not foetal loss [12]. The consequences of laparoscopic surgery in pregnancy have been looked into by small studies. In the short-term period, researchers have found no increase in foetal distress or demise, with no use of tocolysis. In the long term, the resultant children were monitored, with no evidence of any developmental or physical abnormalities detected during the study period [13].
Complications and limitations of laparoscopy

Despite favourable reports of laparoscopic surgery in pregnancy, it is associated with limitations and potential complications.

\section{Entry technique}

Inadvertent introduction of the Veress needle into the gravid uterus with subsequent pneumoamnion has been reported and was a catastrophic complication in a midtrimester pregnancy [14]. To avoid such a complication, the left hypochondrium point (Palmer point) can be used. The technical difficulty of laparoscopic surgery due to the gravid uterus is another factor to be taken into consideration. The primary trocar should be inserted after determining the height of the uterine fundus. Open (Hasson) technique is often suggested in order to avoid trauma to the uterus. Supraumbilical, subxiphoid midline, or left upper quadrant insertion points are used, or umbilical insertion towards the upper abdomen [7, 15], Secondary trocars should be inserted under direct view and higher than they would be in a nonpregnant woman. Open-entry technique can be used to avoid any potential trauma caused by the Veress needle or primary trocar. The use of a special Veress needle incorporating a fibre-optic scope for direct vision on entry is available, providing comfort and confidence to the surgeon in avoiding intraabdominal injury.

\section{The procedure itself}

In most cases the adnexal mass is found in the pouch of Douglas. Dexterity and good exposure are necessary to bring the mass to its original position by using graspers. To prevent damage to the adnexal vessels, only the ovarian ligaments should be pulled up. Using two atraumatic graspers, cystectomy can then be performed. Cystectomy or oophorectomy can be performed as in a nonpregnant woman. Disposable instruments may need to be used, such as rotating graspers/scissors with more than 45-degree angles; ultrasonic scissors or bipolar cutting scissors may aid the operator's comfort and efficiency.

\section{Uterine perfusion and intraabdominal pressure}

In the early stages of introducing laparoscopic management as a surgical option for abdominal pathology in pregnancy, the potential risk of decreased uterine blood flow secondary to the increase in intraabdominal pressure and the theoretical risk of carbon dioxide absorption to both mother and foetus were concerns that were taken into account [5]. Limited experimental data have so far shown that the increased intraabdominal pressure with carbon dioxide appears to have no harmful effects on lamb foetuses in utero [16]. Achieving excessively high intraabdominal pressures is not necessary to gain 
satisfactory port entry in laparoscopy. Pressures of between 11 and $14 \mathrm{mmHg}$ are perfectly satisfactory [7]. Gasless laparoscopy has been used with and without general anaesthesia [17] and has also been shown to be equally effective in achieving good treatment outcomes compared with laparotomy [18]. Partial left decubitus positioning can be used to avoid compression of the uterus on the vena cava, which could decrease placental blood flow.

\section{Malignant ovarian tumours}

When an ovarian tumour is benign, optimal management can be achieved with elective laparoscopic surgery [19]. There has been no report of malignant ovarian cases discovered via laparoscopic surgical management in the 2nd or 3rd trimester of pregnancy. Should malignant disease be suspected, laparotomy is still the choice of access when considering the risk of cyst rupture and spillage during manipulation of the ovary, which in turn risks seeding malignant cells in the peritoneal cavity.

\section{Large ovarian cysts}

Laparoscopic surgery has also been used for significantly large ovarian cysts, and despite that, the median operating time varied from 37.5 [19] to $84 \mathrm{~min}$ [20], and the average hospital stay for postoperative recovery was 2 days [20]. In cases in which large ovarian cysts were found in pregnant obese patients, modifications have been tried by adding an extracorporeal component. The cyst was located laparoscopically, its fluid aspirated to decompress it, and one of the port sites enlarged to $3 \mathrm{~cm}$ for an extracorporeal oophorectomy or cystectomy to be performed. This modification required significantly less carbon dioxide insufflation time and shorter operation time compared with traditional laparoscopic approaches, in turn theoretically reducing any adverse risk to the foetus [21].

\section{Third trimester}

Laparoscopy has also been reportedly used at 24 34 weeks' gestation with good results [6]. Even with emergency cases, reports have described successful laparoscopic management of a torted ovarian cyst in the 3rd trimester [22]. Under expert hands, the laparoscopy can have spectacular applications, as in the case of successful laparoscopic management of a torted ovarian cyst at 25 weeks' gestation in a twin pregnancy [23].

Limitations in the available evidence

Most reports describe a 48-h hospital stay, no use of tocolysis, and no immediate complications to the mother or foetus. Most of the references described in relation to laparoscopy in pregnancy have been either small studies, case reports, or even retrospective analysis of a large number of cases in specialized centres worldwide - hence the limitations when attempting a meta-analysis. Some references have included operations performed under a different context (cholecystectomy), with variation in techniques, instruments, and operator experiences. It is acknowledged that there will be some laparoscopic cases that end up being converted to laparotomy, which may possibly limit the interpretation of the overall efficacy of minimal access surgery. Further limitations that might determine the efficacy of laparoscopic surgery include the operator-dependent factor. Surgical units that specialise in laparoscopic surgery are said to have a more favourable management outcome [19]. Most reports in the literature come from centres and surgeons with special interests, experiences, and skills in laparoscopy. Their results may not reflect the general complication rates [5].

\section{Conclusions}

Laparoscopic gynaecological surgery appears to be safe during pregnancy, although prospective controlled studies and national registries encompassing larger numbers of cases are needed to assess the definitive rate of adverse effects [10]. Nevertheless, increasing interest in laparoscopic treatment for ovarian tumours, particularly in the 1st and 2nd trimesters, is leading to more reports of cases with favourable outcomes.

The pregnant woman may require nonobstetrical surgical operations and interventions. Pregnancy itself can delay the diagnosis, resulting in advanced or acute presentations. Reports have shown that in surgical nongynaecological interventions that commonly occur in the 1st and 2nd trimesters of pregnancy, the risk of miscarriage, premature labour, or foetal demise appears to be related to the underlying pathology, independent of the operative technique. Avoidance of a large laparotomy wound, reduction in postoperative pain and infection risk, quicker restoration to full mobility, and shorter hospital stay may favour the use of a laparoscopic approach in selected pregnant patients.

\section{References}

1. Chung A, Birnbaum SJ (1973) Ovarian cancer associated with pregnancy. Obstet Gynecol 41(2):211-214

2. Munnell EW (1963) Primary ovarian cancer associated with pregnancy. Clin Obstet Gynecol 30:983-993

3. Munkarah AR, Morris R (2001) Malignant disease in pregnancy. High-risk pregnancy management options, 2nd edn. WB Saunders, London, p 948

4. Hutton J (2001) Gynecological disease non-malignant. Highrisk pregnancy management options, 2nd edn. WB Saunders, London, pp 934-935

5. Fatum M, Rojansky N (2001) Laparoscopic surgery during pregnancy. Obstet Gynecol Surv 56(1):50-59 
6. Geisler JP, Rose SL, Mernitz CS, Warner JL, Hiett AK (1998) Non-gynecologic laparoscopy in second and third trimester pregnancy: obstetric implications. JSLS 2(3):235-238

7. Al-Fozan H, Tulandi T (2002) Safety and risks of laparoscopy in pregnancy. Curr Opin Obstet Gynecol 14(4):375-379

8. Oelsner G, Stockheim D, Soriano D, Goldenberg M, Seidman DS, Cohen SB, Admon D, Novikov I, Maschiach S, Carp HJ, Anderman S, Ben-Ami M, Ben-Arie A, Hagay Z, Bustan M, Shalev E, Carp H, Gemer O, Golan A, Holzinger M, Beyth Y, Horowitz A, Hamani Y, Keis M, Lavie O, Luxman D, Oelsner G, Stockheim D, Rojansky N, Taichner G, Yafe C, Zohar S, Bilanca B (2003) Pregnancy outcome after laparoscopy or laparotomy in pregnancy. J Am Assoc Gynecol Laparosc 10(2):200-204

9. Reedy MB, Galan HL, Richards WE, Preece CK, Wetter PA, Kuehl TJ (1997) Laparoscopy during pregnancy. A survey of laparoendoscopic surgeons. J Reprod Med 42(1):33-38

10. Soriano D, Yefet Y, Seidman DS, Goldenberg M, Mashiach S, Oelsner G (1999) Laparoscopy versus laparotomy in the management of adnexal masses during pregnancy. Fertil Steril 71(5):955-960

11. Conron RW Jr, Abbruzzi K, Cochrane SO, Sarno AJ, Cochrane PJ (1999) Laparoscopic procedures in pregnancy. Am Surg 65(3):259-263

12. Visser BC, Glasgow RE, Mulvihill KK, Mulvihill SJ (2001) Safety and timing of nonobstetric abdominal surgery in pregnancy. Dig Surg 18(5):409-417

13. Rizzo AG (2003) Laparoscopic surgery in pregnancy: longterm follow-up. J Laparoendosc Adv Surg Tech A 13(1):11-15

14. Friedman JD, Ramsey PS, Ramin KD, Berry C (2002) Pneumoamnion and pregnancy loss after second-trimester laparoscopic surgery. Obstet Gynecol 99(3):512-513
15. Canis M, Rabischong B, Houlle C, Botchorishvili R, Jardon K, Safi A, Wattiez A, Mage G, Pouly JL, Bruhat MA (2002) Laparoscopic management of adnexal masses: a gold standard? Curr Opin Obstet Gynecol 14(4):423-428

16. Batallan A, Benifla JL, Panel P, Dorin S, Darai E, Madelenat P (1999) Laparoscopic surgery during the second trimester of pregnancy: indications, technique, and fetal repercussions. Report of nine cases and review the literature. Ann Chir 53(4):285-290

17. Tanaka H, Futamura N, Takubo S, Toyoda N (1999) Gasless laparoscopy under epidural anesthesia for adnexal cysts during pregnancy. Reprod Med 44(11):929-932

18. Akira S, Yamanaka A, Ishihara T, Takeshita T, Araki T (1999) Gasless laparoscopic ovarian cystectomy during pregnancy: comparison with laparotomy. Am J Obstet Gynecol 180(3 Pt 1):554-557

19. Yuen PM, Chang AM (1997) Laparoscopic management of adnexal mass during pregnancy. Acta Obstet Gynecol Scand 76(2):173-176

20. Moore RD, Smith WG (1999) Laparoscopic management of adnexal masses in pregnant women. J Reprod Med 44(2):97100

21. Chung MK, Chung RP (2001) Laparoscopic extracorporeal oophorectomy and ovarian cystectomy in second trimester pregnant obese patients. JSLS 5(3):273-277

22. Kim WW, Chon JY, Chun SW, Jeon HM, Kim EK (2000) Laparoscopic procedures during the third trimester of pregnancy. Surg Endosc 14(5):501

23. Bassil S, Steinhart U, Donnez J (1999) Successful laparoscopic management of adnexal torsion during week 25 of a twin pregnancy. Hum Reprod 14(3):855-857 